\title{
Motivación y satisfacción de los trabajadores y su influencia en la creación de valor económico en la empresa*
}

\author{
Alex Medina Giacomozzi** \\ Cecilia Gallegos Muñoz*** \\ Patricio Lara Hadi****
}

\begin{abstract}
Sumario: 1 . Gestión de valor económico y efecto en los trabajadores; 2. Distribución de valor al personal: satisfacción e insatisfacción laboral y motivación; 3. Relación entre la falta de insatisfacción, motivación, satisfacción y creación de valor económico; 4. Conclusiones.
\end{abstract}

Summary: 1 . Economic value management and its effects upon workers; 2. Distribution of value to the employees: satisfaction and dissatisfaction of workers and motivation; 3. Relationship between lack of dissatisfaction, motivation, satisfaction, and creation of economic value; 4 . Conclusions.

Palabras clave: valor económico; motivación; satisfacción; falta de satisfacción; retribución; evaluación laboral.

KEY WORDs: economic value; motivation; satisfaction; lack of satisfaction; salary; labor evaluation.

La gestión del valor económico implica que la dirección de la empresa debe preocuparse de forma eficaz en como alcanzar esta eficiencia en la generación del valor y en como lograr su equitativa distribución, ya que la sociedad actual no puede ser concebida solamente como un mercado sino que está constituida por un conjunto de personas que participan de forma cooperativa y competitiva en la comunidad,

\footnotetext{
* Artículo recibido en mar. y aceptado en sept. 2008.

** Doctor en finanzas. Magíster en administración y dirección de empresas. Profesor asociado Facultad de Ciencias Empresariales, Universidad del Bío-Bío, Chile. Dirección: Av. Brasil s/n, Chillán, Región del Bío-Bío, Chile. E-mail: alex@ubiobio.cl.

*** Magíster en contabilidad y auditoria. Profesor asistente Facultad de Ciencias Empresariales, Universidad del Bío-Bío, Chile. Dirección: Av. Brasil s/n, Chillán, Región del Bío-Bío, Chile. Email: cecilia@ubiobio.cl.

***** Psicólogo. Dirección: Av. Libertad, 887, Chillán, Región del Bío-Bío, Chile. E-mail: plarahadi @hotmail.com.
} 
que tienen determinadas motivaciones y que actúan colectivamente, coordinando sus acciones según los objetivos de sus empresas, en la búsqueda de alcanzar sus propias expectativas, por lo que al centrar nuestro interés en los trabajadores de la empresa surgen dos variables de análisis y que están directamente relacionadas con la motivación, independientes en su génesis, pero que interactúan entre sí, por un lado la falta de insatisfacción laboral, y por otro la satisfacción laboral, aspectos que son analizados en este artículo y su influencia en la creación de valor económico en la empresa. Es fundamental que la empresa identifique y conozca las expectativas de cada uno de sus trabajadores, con lo cual se podrá establecer estrategias que le permitan poder alinear éstas a los objetivos organizacionales y a la creación o incremento de su valor económico, y así tender a reducir la falta de insatisfacción y apuntar a la motivación y satisfacción del personal.

Motivation and satisfaction of the workers and their influence in creating economic value in business

Economic value management implies that management should be interested in reaching an efficient generation of value and its fair distribution. Our society cannot be conceived only as a market because it is constituted by a community of people who participate competitively and cooperatively. They have their own motivations and act collectively by coordinating their actions according to the objectives of their businesses so as to fulfill their expectations. While centering our interest in the workers, two variables of analysis emerge. These variables are directly related to motivation and independent in their genesis, although there is interaction between them: the lack of satisfaction at work and job satisfaction. Both aspects are analyzed in this paper and their influence in the creation of economic value in business. In this scenario, it is fundamental that the firm identify and know the expectations of each one of its workers. This will allow to establish strategies to harmonize these expectations with the organizational objectives and the creation of economic value. This type of initiative tends to reduce the lack of satisfaction and to improve personal motivation.

\section{Gestión de valor económico y efecto en los trabajadores}

La sociedad actual no puede ser concebida solamente como un mercado sino que está constituida por un conjunto de personas que participan de forma cooperativa y competitiva en la comunidad, que tienen determinadas motivaciones y que actúan colectivamente, coordinando sus acciones según los objetivos de sus empresas, en la búsqueda de alcanzar sus propias expectativas.

De lo recién expresado surge una variedad de planteamientos, pero de todos ellos y de acuerdo al enfoque de este artículo solo se hará referencia a 
aquellos conceptos que permitan una mejor comprensión de la relevancia de la gestión del valor.

Un primer aspecto a considerar en la gestión del valor, es el reconocimiento de que la empresa es un ente fundamental dentro de la actividad económica, en la que participan interrelacionadamente diversos agentes económicos, entre los que se destacan los financiadores, usuarios y trabajadores.

Otro aspecto a tener en cuenta y que nace de esta actividad económica, es que el motivo que explica el comportamiento de estas personas como agentes económicos es el valor económico y que este valor, ${ }^{1}$ por ende, depende de las expectativas de estos agentes.

Este valor, por lo tanto, es eminentemente subjetivo, ya que en la medida que representan opiniones de personas determinadas y ésta se individualice en un sujeto particular, será posible proceder a su estimación. Bajo este prisma, según la naturaleza del valor, se entiende éste como una valoración subjetiva, en contraposición a ser entendida como una valoración objetiva (Jaensch, 1974).

Esta estimación del valor implica utilizar determinados subrogados de valor como parámetros de medida, ya sea de carácter cuantitativo (precio, costo, métodos de valorización de empresas, por citar algunos) o cualitativo (satisfacción de las personas).

Ahora, hay que considerar que desde una perspectiva lógica, toda generación de valor lleva implícita su distribución, ya que todo bien puede y debe ser analizado desde el ángulo de su creación y de su apropiación, tal como se describe en la siguiente ecuación.

$$
\mathrm{VE}_{\mathrm{C}}=\mathrm{VE}_{\mathrm{D}}
$$

Siendo:

$\mathrm{VE}_{\mathrm{C}}=$ valor económico creado;

$\mathrm{VE}_{\mathrm{D}}=$ valor económico distribuido.

Lo anterior nos lleva a que esta creación y distribución del valor sean dos facetas de una misma realidad y que no conviene confundir, ya que si bien ambos deben estar en equilibrio, cada una de ellas está sujeta a sus propias características y particularidades (Medina, 2002).

\footnotetext{
${ }^{1}$ No es de extrañar que para Bunge (1982), desde un punto de vista económico, el concepto de valor sea oscuro y confuso ya que, en palabras de este autor, se lo define de diversas formas, ya sea "en términos de necesidades, otras en términos de demanda o como trabajo socialmente necesario".
} 
Naturalmente, al considerar esta igualdad entre creación y reparto del valor, cualquier alteración en la generación afecta en la misma medida a la distribución, no obstante cualquier alteración en la forma como se realiza la distribución no necesariamente va a afectar a la creación. ${ }^{2}$

Esta gestión del valor conduce, por lo tanto, a que la empresa gracias a su actividad procure el aumento del bienestar social de la comunidad en la que se inserta, lo que incluye a uno de sus principales agentes económicos, como son sus trabajadores.

El incorporar en la administración de empresas este modelo de gestión del valor, implica que la dirección de la entidad debe preocuparse de forma eficaz en cómo alcanzar esta eficiencia en la generación del valor y en cómo lograr una distribución equitativa de este valor creado, lo que nos lleva a comentar ambos puntos de vista.

En cuanto al valor económico creado, este nace de la comparación del valor económico de la empresa en un momento del tiempo (por ejemplo año 2) con el obtenido en un momento anterior (por ejemplo año 1), en donde para que se cumpla esta generación de valor, debe darse la condición que se expone en la ecuación (2).

$$
\mathrm{VE}_{\mathrm{C}}=\mathrm{VE}_{\mathrm{F}}>\mathrm{VE}_{\mathrm{I}}
$$

Siendo:

$\mathrm{VE}_{\mathrm{C}}=$ valor económico creado;

$\mathrm{VE}_{\mathrm{F}}=$ valor económico final de la empresa;

$\mathrm{VE}_{\mathrm{I}}=$ valor económico inicial de la empresa.

Al valorar una empresa en su conjunto, a diferencia de valorarla como un bien singular (Franch, 1990), puede hacerse de acuerdo a una variedad de métodos susceptibles de utilizar, por lo que la elección del método dependerá de los objetivos que se persiguen con la valoración, sin olvidar que solo tiene sentido la estimación del valor subjetivo de la empresa (Gomez-Bezares y Santibañez, 1997) y bajo el contexto de la teoría de la entidad (Medina y Gallegos, 2003a; 2003b).

\footnotetext{
${ }^{2}$ En todo caso, este cambio en la forma de reparto podría alterar la equidad de esta distribución y, este hecho, puede llegar a afectar la generación del valor como consecuencia de la pérdida de interés y motivación por parte de los agentes participantes.
} 
En este sentido, la estimación del valor de la empresa implica considerar dos aspectos fundamentales, como son los objetivos que se persiguen con la valoración y los métodos posibles de utilizar.

El objetivo de la valoración puede estar referido a la determinación del valor como parámetro base en una negociación, ya sea de fusión, escisión, compra etc., de una empresa.

No obstante, cualquiera que sea el motivo de la valoración, en definitiva lo que se busca valorar es la capacidad de la dirección para incrementar la riqueza de la empresa. Este hecho hace difícil establecer el valor ideal de la empresa y más complejo aún es escoger el método adecuado para estimar dicho valor (Pisón, 2001).

Si bien existe una amplia variedad de métodos susceptibles de utilizar para la valorización de empresas, la utilidad y connotación de cada uno de estos es distinta, lo que obliga a ser muy cuidadosos en la elección del método, ya que de esto dependerá la validez y aplicabilidad de los resultados obtenidos con la valoración.

Una serie de métodos y clasificaciones aparecen en diversos textos y publicaciones, entre las que se pueden citar, a modo de ejemplo: Aeca (1996), Fernández (2000, 1999), Adserà y Viñolas (1997), Pisón (2001), Caballe (1994).

Es importante indicar, que el método de valorización de empresas a utilizar debe resaltar un aspecto fundamental y que debe ser el norte de toda organización, cual es la gestión del valor económico de la misma (Rappaport, 1998), lo que determina la preeminencia de aquellos métodos de valorización que permitan estimar este valor creado por la entidad, en contraposición de aquellos otros métodos que no lo permiten.

Por tanto, para llegar a determinar que métodos son los más adecuados para la estimación del valor creado se debe tener en consideración tres elementos determinantes del valor, como son la rentabilidad económica esperada, el tiempo en que esta se pueda mantener y el riesgo inherente a esta rentabilidad económica esperada (Martín y Petty, 2001), por lo que en este contexto los métodos basados en el flujo efectivo son los únicos que cumplen estas tres condiciones.

Este mismo planteamiento es sustentado por destacados autores, entre los que se pueden citar a Copeland, Koller y Murrin (2004), Stewart (2000), Rappaport (1998), López (1999), Gómez Bezares y Santibañez (1997), Koller y Mateache (1997), Martín y Petty (2001), Adserà y Viñolas (1997), Stern, Shiely y Ross (2002) etc., por lo que en este contexto destaca el método del flujo efectivo de libre disposición (FEL - conocido como FCF, por su sigla 
en inglés, free cash flow), que es un término acuñado por Copeland, Koller y Murrin (2004) en su propuesta metodológica para la valoración de empresas y el método del valor económico añadido (VEA), que es una propuesta metodológica de Stewart (2000).

Ambos métodos cumplen con las condicionantes del valor, sin embargo el método FEL permite determinar el flujo generado y distribuido a diferencia del método VEA, que solamente permite determinar el flujo generado. En este contexto, es más apropiado para analizar el efecto de la motivación y satisfacción del personal en la generación de valor económico en la empresa, el método FEL.

Desde el punto de vista de la distribución, por tanto, la dirección deberá tener en cuenta a la sociedad, sus valores, creencias y cultura para fijar criterios equitativos de reparto que estimulen, motiven y satisfagan a cada uno de los agentes económicos involucrados, es decir, los financiadores, usuarios y trabajadores. Por tanto, al incorporarlos a la igualdad fundamental expresada en la ecuación (1), obtenemos la siguiente identidad.

$$
\mathrm{VE}_{\mathrm{C}}=\mathrm{VED}_{\mathrm{U}}+\mathrm{VED}_{\mathrm{P}}+\mathrm{VED}_{\mathrm{F}}
$$

Siendo:

$\mathrm{VED}_{\mathrm{U}}=$ valor económico distribuido a los usuarios;

$\mathrm{VED}_{\mathrm{P}}=$ valor económico distribuido al personal;

$\mathrm{VED}_{\mathrm{F}}=$ valor económico distribuido a los financiadores.

\section{Distribución de valor al personal: satisfacción e insatisfacción laboral y motivación}

Al centrar nuestro interés en la distribución de valor a los trabajadores en función de las expectativas que estos tienen, surgen dos variables de análisis y que están directamente relacionadas con la motivación, independientes en su génesis, pero que interactúan entre sí, por un lado la falta de insatisfacción laboral, y por otro la satisfacción laboral.

Es importante recalcar que cuando se cuenta con personal motivado existe mayor probabilidad de aportar al incremento del valor económico, por lo que es fundamental analizar estas dos variables.

En este punto nos encontramos con una variedad de autores que tratan sobre la motivación del personal, entre estos se destaca Maslow (1991) con su teoría de jerarquía de las necesidades, cuyo aporte es fundamental para comprender estas necesidades en relación con las expectativas de los individuos. 
Otros autores destacados son McGregor (1960), con su teoría X-Y sobre el comportamiento humano en el trabajo, e McClelland (1989), con su planteamiento de la teoría de la motivación en base a las necesidades (necesidades de logro, poder y afiliación).

A los autores anteriores, en épocas más recientes se agregan los planteamientos de Vroom (1979), quien plantea que la motivación es producto de multiplicar tres factores (valencia, expectativa e instrumentalidad), y de Shein (1991) sobre teoría del hombre complejo.

También es interesante lo planteado por Adams (1997), quien considera que a los trabajadores además de interesarles la obtención de recompensas por su desempeño, también desean que estas sean equitativas, lo que transforma en mas compleja la motivación, es decir, existe una tendencia a comparar los aportes (esfuerzo) y resultados (recompensas), pero además a realizar comparaciones con otras personas ya sea de la organización o no.

También se destaca el aporte de Herzberg (1959), quien propone una teoría en donde la satisfacción laboral dependerá del grado de satisfacción de dos factores.

Siguiendo a Herzberg, el primero de estos factores agrupa variables que se relacionan o localizan en el ambiente que rodea a las personas y abarcan las condiciones en que desempeñan su trabajo. Como estas variables son administradas y decididas por la empresa, se encuentran fuera del control de los trabajadores. Las principales variables aisladas serían: salario, beneficios sociales, tipo de dirección o supervisión, condiciones físicas y ambientales de trabajo, políticas y directrices de la empresa, el clima de las relaciones entre los directivos y las personas que trabajan en la empresa, los segmentos internos, estatus, prestigio etc.

Históricamente o tradicionalmente solo estas variables (higiénicas) fueron tomadas en cuenta en la motivación de los empleados. En este sentido para lograr que las personas trabajen más se apelaba a premios, mejoras salariales, incentivos de distinta índole, liderazgo democrático, políticas empresariales más abiertas y estimulantes, es decir, incentivos externos a cambio de un mayor trabajo.

Según las investigaciones de Herzberg (1959) cuando los factores higiénicos son óptimos, solo evitan la insatisfacción de los empleados, pues no consiguen elevar consistentemente la satisfacción, y cuando lo hacen no es por un tiempo prolongado. No obstante, cuando estos factores son precarios o pésimos provocan directa insatisfacción.

A causa de esa influencia más orientada a evitar la insatisfacción, denominados factores higiénicos por Herzberg (1959), tenemos que estos factores 
son esencialmente profilácticos y preventivos, lo que hace que no necesariamente provoquen satisfacción.

Existiría un segundo grupo de variables y factores que se encontrarían relacionados más directamente con la satisfacción de la persona y que tienen relación con la naturaleza de las tareas que el individuo ejecuta. A estos factores Herzberg (1959) los llama motivacionales o intrínsecos. Estos factores están bajo el control del individuo, pues se relacionan con lo que él hace y en lo que se desempeña. Estos factores involucran los sentimientos relacionados con el crecimiento y el desarrollo personal, el reconocimiento profesional, las necesidades de autorrealización, la responsabilidad y dependen de las tareas que la persona realiza en su trabajo.

Tradicionalmente las tareas y cargos han sido diseñados y definidos en términos de eficiencia y economía y han desestimado aspectos como el reto y oportunidad de creación individual, con esto se pierde el significado psicológico para la persona que lo ejecuta teniendo un efecto "des motivador" que provoca apatía, desinterés y falta de sentido ya que la empresa solo ofrece un lugar cómodo de trabajo.

Según las investigaciones de Herzberg (1959), el efecto de los factores motivacionales sobre el comportamiento de la persona es mucho más estable y profundo y cuando son óptimos provocan satisfacción de las personas, pero cuando son precarios más que insatisfacción provocan su evitación. A estos factores Herzberg los llama también factores de satisfacción.

Dentro de este marco, los factores responsables de la satisfacción profesional de las personas están totalmente desligados y son distintos de los factores que originan la insatisfacción profesional, para Herzberg (1959) el opuesto de la satisfacción profesional no sería la insatisfacción, sino ninguna satisfacción profesional, así mismo el opuesto de la insatisfacción laboral o profesional sería ninguna insatisfacción laboral, y no la satisfacción como es de suponer.

A partir de diversas investigaciones se concluye que eliminando la característica de descontento de un puesto no necesariamente se provoca la satisfacción en el mismo. A partir de esto se supone la existencia de un continuo dual donde lo opuesto de satisfacción es no satisfacción y el opuesto de descontento, no descontento.

De acuerdo a lo anterior, los factores que conducen a la satisfacción en el trabajo son independientes y diferentes a los que conducen al descontento, por lo tanto los administradores que buscan eliminar factores que crean descontento pueden traer armonía, pero no necesariamente motivación. Estos administradores solo apaciguan la fuerza laboral en lugar de motivarla. Cuando 
estos factores están dados óptimamente las personas no están descontentas, sin embargo tampoco satisfechas. Para motivar a la persona Herzberg (1959) sugirió poner énfasis en los motivadores (factores que aumentan la satisfacción en el trabajo). En este sentido el énfasis debería estar dado en modificar el contenido mismo de la tarea como fuente de satisfacción laboral.

Dado todo lo anterior, podemos afirmar que un elemento central en toda gestión del valor económico en la empresa está en sus trabajadores y en la forma en que estos se motivan, entendiendo que la motivación se refiere al impulso y esfuerzo por satisfacer un objetivo o expectativa, lo que indica que claramente está asociado a la eficacia de la actuación del trabajador y, por consecuencia, su directa relación con la eficacia organizacional. La satisfacción, en cambio, se refiere al gusto que se experimenta una vez cumplido el objetivo o expectativa, en otras palabras, la motivación implica un impulso al resultado, mientras la satisfacción es el resultado ya experimentado.

De lo anterior se deduce que un trabajador podría disfrutar de una alta satisfacción en su trabajo, pero contar al mismo tiempo con un bajo nivel de motivación.

\section{Relación entre la falta de insatisfacción, motivación, satisfacción y creación de valor económico}

El personal constituye un capital humano fundamental para la organización, por lo que es de suma importancia que estos perciban que su trabajo en la empresa les representa para ellos expectativas de generación de valor, es decir, estar satisfechos, sentir motivación y falta de insatisfacción.

Comprender estas expectativas por parte de la empresa permitirá sentar las bases para que la organización esté en condiciones de crear valor económico.

Conceptualmente las expectativas están constituidas por el deseo o conjunto de deseos que determinan nuestras actitudes, creencias y condicionan nuestro comportamiento y percepción de las cosas, por lo que estas pueden ser ideales, predecibles, normativas o inexistencia de expectativas.

Ahora resulta relevante distinguir entre aquellas expectativas asociadas a la actividad laboral de aquellas otras expectativas no laborales, siendo las primeras las que son objeto de este estudio, aunque debemos reconocer que en la realidad es muy difícil poder separar una de otra.

Al centrar el análisis en las expectativas laborales, es fundamental que la empresa identifique y conozca las expectativas de cada uno de sus trabajadores, con lo cual se podrá establecer estrategias que le permitan a la direcci- 
ón poder alinear estas a los objetivos organizacionales, ya que de esta forma se puede reducir la falta de insatisfacción y apuntar a la motivación y satisfacción del personal.

Para lograr y mejorar el nivel de motivación del personal, debe generarse una coincidencia entre el logro de las expectativas que ellos se han cifrado y los resultados que obtienen con su trabajo en la empresa.

Esto obliga a la organización a conocer y comprender estas expectativas para así responder adecuadamente a cada una de ellas. En este sentido, habrá personas que sus mayores expectativas se expresan en términos monetarios, pero otros tendrán otras prioridades.

La actividad laboral desempeñada por los trabajadores implica, por un lado, que la empresa sea capaz de identificar aquellos factores que provocan insatisfacción laboral y, por otro, aquellos factores que motivan al personal y que, por tanto, generan satisfacción en ellos.

\section{Falta de insatisfacción}

Para alcanzar esta falta de insatisfacción por parte del trabajador, la empresa debe preocuparse principalmente del ambiente de trabajo, del clima laboral en la organización y de los servicios y beneficios al personal.

v Condiciones de trabajo. Si bien esto puede ser considerado una condición básica de la actividad laboral, en la medida que la construcción, espacios físicos, maquinarias, mobiliario y todo aquello que se requiera para el desarrollo de las actividades laborales sean adecuado y tengan en consideración la salud física y psíquica del individuo, permitirá una mejor calidad de vida "laboral".

- Clima laboral. En la medida que el ambiente de trabajo sea grato, como las relaciones entre el personal y de este con los superiores sea de mutua colaboración y apoyo, se tendrán las condiciones necesarias para un buen clima laboral.

- Servicios y beneficios al personal. Son programas que incluyen un amplio grupo de alternativas que van en directo beneficio de los trabajadores, entre los que se pueden encontrar, a modo de ejemplo, una jornada laboral menor a la legal, servicios (ayuda supermercado, planes financieros, comedores, traslado etc.) o protección (atención médica, seguros de vida etc.), todo lo cual es un complemento que mejora la "calidad" de vida laboral y familiar del trabajador. 
En síntesis, estos factores ayudan a que el trabajador sienta una falta de insatisfacción laboral como consecuencia de la preocupación de la empresa por el contexto laboral en que este se desempeña y por ser considerado como parte activa y fundamental dentro de la organización.

\section{Motivación y satisfacción}

Para motivar y tender a la satisfacción del personal, se debe, en primer lugar, reconocer los aportes individuales que cada persona realiza en la empresa, por lo que es necesario evaluar al trabajador en su actividad laboral, ya que esto permitirá conocer su desempeño y comportamiento y como consecuencia de ello recibir una retribución consistente con el resultado de esta evaluación.

En este contexto, los propósitos perseguidos en todo proceso de evaluación se circunscriben (Sartain, 2006) en obtener información sobre la actividad del personal que permita garantizar el logro de los objetivos de la empresa y el aporte que cada trabajador ha realizado al logro de estos objetivos.

Esta información sobre la actividad del personal permitirá conocer el rendimiento del trabajador, por lo que la evaluación aportará antecedentes que lleven a estrategias para su mejora, lo que implica tomar decisiones sobre capacitación y formación, como también sobre gestión de carreras, reclutamiento, relaciones con el personal, en definitiva decisiones sobre estrategias de desarrollo de recursos humanos.

Es importante indicar que toda evaluación implica considerar tres aspectos fundamentales en este proceso (Robbins, 1999), como son los resultados de las actividades individuales, las conductas y los rasgos personales, en donde cada uno de ellos tendrá una ponderación diferente de acuerdo a la situación a evaluar.

Toda evaluación debiera tener como consecuencia una retribución acorde a los resultados obtenidos, por lo que esta retribución al personal tiene por objetivo alcanzar la eficiencia y equidad, partiendo de una condición básica que es el cumplimiento de la legalidad.

Esta eficiencia implica que la retribución debe estar en concordancia con el incremento en la productividad o en el control de los costos laborales, en la búsqueda por alcanzar los objetivos organizacionales.

El sistema de retribución, desde el punto de vista de la equidad (García y Casanueva, 1999), debe reconocer tanto las contribuciones del personal como sus necesidades. En el caso de las contribuciones, se entenderá que se debe ofrecer una mayor retribución por un rendimiento mayor o bien, por la mayor experiencia o formación. 
Complementariamente a lo anterior, las necesidades también deben ser consideradas en la retribución, lo que implica reconocer que las personas requieren de un estipendio que les permita vivir adecuadamente y cumplir, al menos, con sus necesidades básicas.

Este reconocimiento de la identificación de la contribución individual, partiendo del cumplimiento de las necesidades básicas, es un hecho fundamental a la hora de retribuir al personal (Chiavenato, 1999), ya que toma en consideración las particularidades de cada uno, en su propia realidad y expectativas.

Por tanto, la motivación y satisfacción del personal se da en la medida que se reconoce y retribuye el aporte individual a la organización que cada trabajador ha realizado, en donde este aporte está dado por todo aquello que recibe como consecuencia de la eficiencia y contribución que este ha tenido en la entidad.

Ahora, esta retribución debe estar en concordancia con las expectativas laborales de los individuos y ser consistente en el tiempo, por lo que la forma de retribución debe considerar esta complejidad del ser humano e incorporar aquellos aspectos que estén en armonía con sus valores.

Si nos centramos en las formas de retribución al personal, estas pueden revestir una variedad de formas y, que en términos generales, podemos clasificar en dos grandes áreas, por un lado la retribución no económica y, por otro, la retribución económica.

En lo que respecta a la retribución no económica, esta está asociada al reconocimiento dado al trabajador por su productividad, comportamiento o actitud y que puede revestir una variedad de formas, el que puede ser de carácter formal o informal, siendo el primero el que mejor refleja la importancia del reconocimiento y es una excelente forma de valorar al trabajador por su participación en la empresa.

En cuanto a la retribución económica, este es uno de los incentivos fundamentales para motivar al personal en el desempeño de su actividad laboral y permite establecer de forma cuantitativa y clara el aporte que cada cual recibiría por su eficiente y eficaz participación en las actividades de la empresa, por lo que esta reviste diversas modalidades de participación económica que la entidad puede implementar: fija, variable o mixta.

En todo caso, esta retribución económica parte del parámetro básico que es la remuneración, de acuerdo al sueldo o estipendio que las partes hubieren acordado y que, a su vez, debe estar en consonancia con las disposiciones legales que regulan esta relación laboral. 
- Retribución económica fija. En este caso, el trabajador recibe un sueldo fijo por cada período de tiempo, el cual puede incorporar la contribución de cada cual, pero no permite reconocer en esta modalidad la eficiencia aportada por la persona.

- Retribución económica variable. En esta opción, el trabajador solamente recibe un estipendio variable sujeto al cumplimiento de terminadas condiciones, por cada período de tiempo (Robbins, 1999), lo cual es muy útil como parámetro de medida de la eficiencia aportada por el trabajador, pero que puede provocar un direccionamiento del esfuerzo personal exclusivamente hacia el logro de esta retribución, perdiendo de vista otros aspectos fundamentales de la vida del ser humano.

- Retribución económica mixta. Sueldo fijo más un sueldo variable sujeto al cumplimiento de terminadas condiciones, por cada período de tiempo.

Al buscar unir la retribución económica a la distribución de valor al personal, nos encontramos que de las tres modalidades vistas la tercera es la que cumple con mayor claridad los objetivos de toda retribución, dentro del marco de las expectativas de los trabajadores, por lo que será esta la base de toda distribución de valor a los trabajadores.

Por tanto, al centrar nuestro interés en la remuneración mixta, en esta se incluye, en distinta proporción, una parte fija (remuneración base) y otra variable. Esta última puede estar constituida por una retribución por mérito y/o una retribución por incentivos.

La retribución por mérito, que es la acción que hace al hombre digno de premio y consiste en entregar al trabajador una cifra de dinero por las buenas acciones realizadas que lo hacen destacar dentro de sus pares. Esta cifra puede ser entregada por una vez o como incremento de la remuneración por un período definido de tiempo. En síntesis, esta forma de retribución permite diferenciar entre las personas, pagando cantidades diferentes en momentos distintos, según el mérito de cada cual, lo cual se constituye en una forma de distribuir valor al personal. Este tipo de retribución, en la realidad recibe distintas denominaciones, como puede ser: bonificación, bono, asignación de mérito etc.

En cuanto a los incentivos, estos unen la retribución económica directamente al rendimiento, ya sea del trabajador, de la unidad o incluso de una unidad estratégica de negocios. En este punto nos encontramos con dos aspectos fundamentales e interdependientes, por un lado, la forma en como puede 
ser definido el rendimiento ${ }^{3} \mathrm{y}$, por otro, los tipos de incentivos ${ }^{4}$ susceptibles de entregar, siendo estos últimos los siguientes:

> Opción de acciones. Es relevante comentar que en la actualidad las opciones de acciones ${ }^{5}$ (stock options), para el caso de sociedades anónimas, es una forma muy apropiada de incentivo a los directivos de las empresas, por lo que es fundamental que este plan de opción de acciones esté supeditado a la creación de valor para el accionista, o sea, que adopte la misma base de estimación y sea ejecutable en el largo plazo. ${ }^{6}$

- Porcentaje sobre beneficios. En este caso el incentivo se determina a partir de un porcentaje de los resultados, que pueden estar determinados a partir del resultado final o parcial (unidad estratégica de negocios, centro de responsabilidad etc.), dependiendo del nivel jerárquico de quien reciba dicho beneficio. En este sentido, mientras mayor sea la jerarquía del trabajador, mayor tendencia a obtener beneficios con esfuerzos globales, en sentido contrario, mientras menor sea la jerarquía, mayor tendencia a obtener beneficios vía esfuerzo individual.

En cuanto a los beneficios finales, estos pueden ser calculados a partir del resultado económico (utilidad del ejercicio) o del resultado financiero (flu-

\footnotetext{
${ }^{3}$ Este rendimiento puede ser medido de diversas formas, como puede ser ahorro en costos, aumento del volumen de producción, consecución de estándares de calidad etc.

${ }^{4}$ Muchas empresas, ya sea por exigencia legal o contractual, entregan a cada uno de sus trabajadores una gratificación consistente en repartir una parte de las utilidades obtenidas en un período, hecho que es muy positivo, pero que en realidad no representa un incentivo para mejorar la actuación, ya que no está relacionado con el desempeño particular de cada trabajador, sino que con los resultados globales obtenidos.

${ }^{5}$ Siguiendo a Hall (2000), existen tres tipos de planes de opciones, a saber:

- Planes de valor fijo, aquí el directivo recibe opciones de un valor predeterminado (ya sea una cantidad de dinero o un porcentaje sobre una base) año tras año, a lo largo de toda la vida del plan.

- Planes de cantidad fija, aquí el directivo recibe una serie de otorgamientos anuales de una cantidad fija de opciones durante el período de vigencia del plan.

- Plan de mega-otorgamiento, aquí el directivo recibe un único otorgamiento de opciones, en lugar de los anuales; el precio de ejercicio y la cantidad de opciones se fijan en el momento de otorgarlas.

${ }^{6}$ De estos métodos de opción de acciones el mas utilizado, según Hall (2000), es el de planes de valor fijo y habitualmente se determina mediante la fórmula de valuación de Black-Scholes, o similares, que pondera varios factores: la cantidad de años hasta que la opción expire, las tasas de interés predominantes, la volatilidad del precio de la acción y la tasa de dividendo de la acción.
} 
jo efectivo), siendo mas aconsejable la última alternativa, ya que minimiza el riesgo de influencia sobre la obtención del resultado económico.

En lo que respecta a los beneficios parciales, estos se pueden determinar a partir de las utilidades de un centro de beneficios o la disminución de costos de un centro de costos.

v Comisión. Este tipo de incentivo se obtiene a través del esfuerzo individual, que lo diferencia del anterior, y se determina como una tasa, porcentaje o guarismo calculado sobre los ingresos.

\section{Conclusiones}

Como cierre de este artículo podemos resaltar algunos aspectos relevantes y que grafican la importancia de una apropiada gestión de la empresa en pos de incrementar el valor económico de la organización y, por ende, de su adecuada distribución a los trabajadores, en la forma de una retribución no económica como económica.

Esta distribución de valor al personal implica considerar a este en su realidad, por ende, debe ser justa y equitativa, de lo contrario, afectará el potencial de creación de valor que la empresa podría generar.

Ser justa implica dar a cada cual según su contribución, es decir, de acuerdo a lo aportado por cada cual a la organización y ser equitativa quiere decir que esta distribución debe ser equilibrada entre todos los miembros de la organización. Para lograr lo anterior hay que considerar que esta retribución debe estar en consonancia con la evaluación del personal.

Esta evaluación es el elemento esencial a la hora de distinguir el aporte entregado por cada trabajador y el reconocimiento de sus cualidades que permitan, a partir de esto, alcanzar una adecuada retribución.

En lo que se refiere a la retribución, es importante recalcar que esta no debe ser mirada exclusivamente como un estipendio monetario, sino que es mucho más amplia y que debe responder a las propias expectativas del trabajador, por lo que la empresa debe ser capaz de identificar aquellos factores que provocan insatisfacción laboral y, por otro, aquellos factores que motivan al personal y que, por tanto, generan satisfacción en ellos.

De acuerdo a lo anterior, los aspectos que permiten alcanzar una falta de insatisfacción del personal están las condiciones de trabajo, el clima laboral y los servicios y beneficios al personal, siendo todas muy relevantes como parte de la distribución del valor económico al personal, por lo que en la medida 
que estas sean adecuadas y tiendan a mejorar se estará en la senda correcta en la consideración del personal de la empresa.

No obstante lo expresado, la retribución es uno de los incentivos fundamentales para motivar al personal en el desempeño de su actividad laboral, en donde esta puede revestir la forma de retribución no económica y retribución económica.

Es importante destacar que la retribución no económica está asociada al reconocimiento dado al trabajador por su productividad, comportamiento o actitud y que puede revestir una variedad de formas, ya sea de carácter formal o informal, siendo el primero el que mejor refleja la importancia del reconocimiento y es una excelente forma de valorar al trabajador por su participación en la empresa.

En lo que respecta a la retribución económica, de las tres modalidades posibles de utilizar, la retribución mixta es la que cumple con mayor claridad los objetivos de toda retribución, que es la eficiencia y la equidad, por lo que en esta se incluye la retribución por mérito y los incentivos, adicional a la remuneración base.

En resumen, en la medida que el trabajador esté motivado, satisfecho y con una falta de insatisfacción estará contribuyendo con mayor eficiencia y eficacia al logro de los objetivos organizacionales, lo cual debiera implicar una mejora en los resultados de la empresa y, en definitiva, en un aporte al incremento en el valor económico de la compañía.

Ahora bien, este incremento en el valor económico en la empresa conlleva automáticamente al aumento del valor económico distribuido a sus agentes fundamentales, es decir, clientes, trabajadores y financistas, por lo que se cumple la igualdad de valor económico creado igual a valor económico distribuido.

Por último, no se debe perder de vista el equilibrio de largo plazo que la distribución del valor económico implica, lo cual debiera llevar a la empresa a gestionar esta distribución equitativamente entre todos sus agentes y, particularmente, con su personal, lo que en definitiva constituye el circulo virtuoso de la gestión del valor económico.

\section{Referencias bibliográficas}

ADAMS, S. El principio de Dilbert. México: Granica, 1997.

ADSERA, X.; VIÑOLAS, P. Principios de valoración de empresas. Bilbao: Deusto, 1997. 
AECA (Asociación Española de Contabilidad y Administración de Empresas). Principios de valoración de empresas: estudio de aplicabilidad de los diferentes modelos de valoración. Madrid: Aeca, 1996.

BUNGE, M. Economía y filosofía. Madrid: Tecnos, 1982.

CABALLE, V. Métodos de valoración de empresas. Madrid: Pirámide, 1994.

CHIAVENATO, I. Administración de recursos humanos. Bogotá: McGraw-Hill, 1999.

COPELAND, T.; KOLLER, T.; MURRIN, J. Valoración, medición y gestión del valor. Bilbao: Deusto, 2004.

FERNÁNDEZ, P. Valoración de empresas. Barcelona: Gestión 2000, 1999. . Creación de valor para los accionistas. Barcelona: Gestión 2000, 2000.

FRANCH, J. Fundamentos del valor económico. Madrid: Unión Editorial, 1990.

GARCÍA, J.; CASANUEVA, C. Gestión de empresas. Madrid: Pirámide, 1999.

GÓMEZ BEZARES, F; SANTIBAÑEZ, J. Cálculo y gestión del valor de la empresa. Boletín de Estudios Económicos, v. 52, n. 162, p. 429-457, dic. 1997.

HALL, B. What you need to know about stock options. Harvard Business Review, Mar./Apr. 2000.

HERZBERG, F. The motivation to work. New York: Wiley, 1959.

JAENSCH, G. Valoración de la empresa. Barcelona: Ariel, 1975.

KOLLER, T.; MATEACHE, P. Impacto y puesta en marcha de una gestión basada en el valor. Boletín de Estudios Económicos, v. 52, n. 162, p. 409-428, dic. 1997.

LÓPEZ, F. La dirección de negocios orientada a la creación de valor. Harvard-Deusto Business Review, n. 89, p. 68-77, mar./abr. 1999.

MARTÍN, J.; PETTY, J. La gestión basada en el valor. Barcelona: Gestión 2000, 2001.

MASLOW, A. Motivación y personalidad. Madrid: Díaz de Santos, 1991.

McCLELLAND, D. Estudio de la motivación humana. Narcisa, 1989.

McGREGOR, D. El lado humano de las organizaciones. Madrid: Debate, 1960.

MEDINA, A. Evaluación de la gestión del valor en la empresa: propuesta de un modelo. Revista Teoría, v. 11, p. 43-50, 2002.

; GALLEGOS, C. Metodología de preparación de flujos de libre disposición a partir de estados finales de contabilidad. Revista Contabilidad y Auditoria, Santiago, 2003a. 
; . Metodología de valorización de empresas por medio del descuento del flujo de efectivo de libre disposición y su aplicación a una empresa real. Revista Horizonte Empresarial, Santiago, 2003b.

PISÓN, I. Dirección y gestión financiera de la empresa. Madrid: Pirámide, 2001.

RAPPAPORT, A. La creación de valor para el accionista. Bilbao: Deusto, 1998.

ROBBINS, S. Comportamiento organizacional. México: Prentice Hall, 1999.

SARTAIN, L. Agente del cambio. Revista Trend Management, Buenos Aires, p. 7073, ago./sep. 2006.

SHEIN, E. Psicología de las organizaciones. Cali: Prentice Hall, 1991.

STERN, J.; SHIELY, J.; ROSS, I. El reto del EVA. Barcelona: Gestión 2000, 2002.

STEWART, G. En busca del valor. Barcelona: Gestión 2000, 2000.

VROOM, V. Motivación y alta dirección. México: Trillas, 1979. 